\title{
24. PHOTOGRAPHIC ASTROMETRY (ASTROMETRIE PHOTOGRAPHIOUE)
}

\author{
PRESIDENT: \\ VICE PRESIDENT: \\ ORGANIZING COMMITTEE:
}

William F. van Altena.

Christian de Vegt.

A. N. Argue, P. Brosche, R. B. Hanson, P. A. Ianna,

D. D. Polojentsev, C. Turon, A. R. Upgren.

\subsection{Introduction}

Commission 24 had seven sessions during the XXIst General Assembly of the IAU in Buenos Aires, one to discuss the business of the Commission and six to report and plan the scientific activities of the Commission members. In addition, numerous members were involved in meetings of the Working Group on Reference Frames (WGRS) and the Joint Discussions on Hipparcos and the Hubble Space Telescope. The highlights of the meeting for many of our members were the reports of the continuing successful observations with Hipparcos. Preliminary results were shown that demonstrated that the accuracy of the positions, parallaxes and photometry were in accord with the predictions for the revised mission. Comparisons were made with ground based observations of the same objects which showed no systematic errors other than those that could be attributed to the ground based positions, parallaxes and photometry. In addition, reports were given on the first astrometric results obtained with the HST, which indicate that high accuracy binary star observations can be made and that the positional accuracy of the Fine Guidance Sensors may be able to reach their design goal, in spite of the optical problems. Further information on both Hipparcos and the HST is contained later in this report and in the Transactions of the two Joint Discussions. Similarly, a full report of the Joint Discussion on Reference Systems can be found in those Transactions.

\subsection{Business Meeting}

The Business Meeting was held during the second afternoon session of July $24 ; 23$ members were present. The first order of business, following the welcoming remarks by the President was the certification of the election results for the officers for 1991 through 1994. President: Chr. de Vegt (Germany); Vice President: C. Turon (France); Scientific Organizing Committee: A. N. Argue (UK), P. D. Hemenway (USA), H. Jahreiss (Germany), D. D. Polojentsev (USSR), J. Stock (Venezuela), W. F. van Altena (USA), J.-J. Wang (PRC). The rules of our Commission state that the Vice President normally assumes the Presidency in the following triennium, and that the Scientific Organizing Committee consists of six members and the outgoing president. The above results of the election for the period 1991 - 1994 were accepted by the members of the Commission present.

The Members observed a minute of silence in respect for two of our members who died during the past triennium: Past President S. Vasilevskis (USA) and member X.-H. Zhou (PRC).

Thirteen new members of the Commission were approved: R. Bouigue (France); W. Dick (Germany); M. Hemenway (USA); I. Kumkova (USSR); D. J. MacConnell (USA); M. Miyamoto (Japan); L. Morrison (UK); W. Osborn (USA); G. Pizzichini (Italy); I. Platais (USSR); P. J. Shelus (USA); T.-g. Yang (PRC); N. Zacharias (Germany). T. Girard (USA) was approved as a Consultant to the Commission and one resignation was received from L. Stange (Germany) who retired recently. This brings our membership in the Commission to 140.

A report from the Working Group on Parallax Standards was given by A. R. Upgren, who reported that the standards are being observed at the parallax observatories that are still active. It was agreed that an inventory of standard fields and plates taken at each observatory should be made and an effort made to begin measurement of the plates. The Working Group will report on the results of the survey and reductions at the next General Assembly.

A brief report from the Working Group on Star Lists was given by T. E. Corbin, who indicated that the bright portion of the list from 9th to 11 th mag. had been prepared at the Royal Greenwich 
Observatory by L. Morrison and the fainter part from 11 th to 13 th mag. had been prepared at the US Naval Observatory. While the bright part was relatively easy to prepare based on the INCA database, considerable difficulties had been encountered in preparing the fainter portion and some gaps in the spatial distribution still exist. More details of this report can be found in the Commission 8 report.

Many problems were encountered by our members in dealing with and obtaining service from the IAU Travel Agent, Marsans. A resolution was therefore passed directing the President to ask the Executive Committee to instruct Marsans to drastically improve its service to the IAU members during the meetings. The President was informed by the Executive Committee that due to the large number of complaints that it had received, such action was being taken.

\subsection{Triennial Report}

W. J. Luyten writes that he is continuing his search for very faint large proper motions stars and low-luminosity objects in the far southern hemisphere on plates taken with the ESO telescopes. His progress has been slow however since he is doing the work with a blink microscope.

\subsection{Poster Papers}

A new feature of this General Assembly'was the institution of "Poster Papers". Three poster papers dealing with the scientific activities of our Commission were presented.

C. E. López displayed some results of his ongoing work on the "Astrometry of Southern Variable and Suspected Variable Stars and Suggestions for a New Format of the Left-Hand Page of the General Catalogue of Variable Stars". As a part of the Yale-San Juan Southern Proper Motion program he has determined improved positions of over 2000 confirmed variable and 500 suspected variable stars from measurements of the first-epoch plates of the SPM. The new positions are referred to the system of the SRS and have an average single coordinate standard error in their positions of $0.7 "$. The region south of declination $-67^{\circ}$ has been completed while about 1300 objects located in Ara, CrA, and northern Pav are being processed; work is now continuing in the region of the South Galactic Pole. He also proposed that the GCVS include a position quality code, and that additional information on improved positions and finding charts in the literature be included.

G. Pizzichini and M. R. Cristallo reported on a search for $x$-ray emission that they have initiated among several hundred Lowell Observatory proper motion stars that lie within the fields observed with the Einstein IPC detector. They hope to add to the number of nearby stars observed for $\mathrm{x}$-ray emission given that a selection of stars based on proper motion will be dominated by nearby stars. In addition they plan to study the number of high velocity stars in the sample that are found to have $\mathrm{x}$-ray emission.

S. Roeser presented a paper on the PPM Catalogue which detailed the characteristics of the 380,000 stars included in the Catalogue. More details about this paper and a companion oral paper on the same subject can be found in the report of Commission 8 for 31 July entitled "Optical Reference Frames and Catalogues - Fainter Extension of the Optical Frame, I"

\section{$\underline{5.0 \text { Scientific Sessions }}$}

At the urging of the General Secretary, Dr. Derek McNally, the commissions were encouraged to hold joint meetings between those commissions with scientific issues of common interest. It was in that spirit that the Presidents of Commissions 8 and 24 decided to hold four of their sessions jointly. In addition, one of the four included members of Commission 26 while another included members of Commission 40 . The organization of so many joint commission meetings was complicated and very time consuming and I would like to express my gratitude to the President of Commission 8, Dr. M. Miyamoto for the enormous effort that he put in to making the meetings a success. Thanks are also due to my colleague Dr. I. Platais, who took over my responsibilities during my absence from New Haven prior to the General Assembly. In order to avoid duplication, the two joint sessions 27 July "Astrometry in the Southern Hemisphere" and 31 July "Optical Reference Frames and Catalogues - Fainter Extension of the Optical Frame, II" are described here while the other two sessions of 29 July "Linking the Optical and Radio Reference Frames" and 31 July "Optical Reference Frames and Catalogues - Fainter Extension of the Optical Frame, I" are described in the Transactions of Commission 8. 


\subsection{July "Progress Reports on Observing Programs and Catalogues" chaired by Chr. de Vegt.}

H. Jahreiss and W. Gliese reported on the status of "The Third Catalogue of Nearby Stars", which is nearing completion at the Astronomisches Rechen Institut. A preliminary version of the CNS3 is now available on a new CD-ROM that has just been released by the NASA Astronomical Data Center. It contains all known stars within 25 parsecs of the Sun, as determined by the 1989 preliminary version of the General Catalogue of Trigonometric Parallaxes now being completed at Yale and a combination of photometric and spectroscopic parallaxes. The CNS3 contains 3804 objects, among which are 552 systems with 1091 components. In addition there are 195 spectroscopic binaries, 45 suspected SB's and 43 objects with variable radial velocity. Every star with a trigonometric parallax greater than 0.039 " has been included, even though other evidence indicates that 505 stars have larger distances. Only photometric parallaxes exist for 1165 stars and for 734 stars the trigonometric parallax was replaced by the photometric parallax. Contrary to the practice of the CNS2 (Gliese 1969), the different types of parallaxes were not combined. Almost half of the stars have parallaxes with a precision better than $15 \%$, while the errors in the absolute magnitudes for most of the remaining stars are on the order of $0.4 \mathrm{mag}$. A comparison of the CNS3 with the CNS2 reveals no significant systematic differences in the parallaxes.

W. van Altena reported on the status of the new edition of "The General Catalogue of Trigonometric Parallaxes" (YPC) which is being prepared at the Yale University Observatory in collaboration with J. T. Lee and E. D. Hoffleit. As with the CNS3, a preliminary version of the YPC has been released on the new CD-ROM issued by the Astronomical Data Center. A new weighting system has been used for the YPC which is based on the root-square-sum of the scaled published error and a constant error for the observatory, or series in question. Of the 15,346 parallaxes for 7,879 stars included in this version, 688 are negative and approximately 1500 are usable for the calibration of stellar masses, distances and absolute magnitudes.

F. Crifo described the Hipparcos Input Catalogue in a paper co-authored by C. Turon. The Input Catalogue was prepared as the observing programme for ESA's highly successful Hipparcos astrometric satellite. It includes 118,000 stars drawn from the lists of many proposers according to their scientific priority and a "survey" (basic list of bright stars), and the operational requirements of the satellite. The INCA consortium has either selected the best data from the published literature, or in the many cases where the data were of low accuracy, set up new ground based observational programs to determine the needed stellar parameters. The printed version of the Input Catalogue is now in press. It includes a brief summary of the selection criteria, and a detailed description of the the stellar content as a function of magnitude, spectral type, duplicity, variability and location on the sky. Identification charts are also given for some 10,000 faint stars, in addition to charts for stars in open galactic clusters and in the Magellanic Clouds. Details on each component of double/multiple systems are also available. A great deal of additional information on the status of the Hipparcos satellite can be found in this volume in the Joint Discussion on Hipparcos.

W. van Altena described a new HST Fixed Head Star Tracker (FHST) catalogue that T.-g. Yang is preparing at the Yale University Observatory. The new FHST catalogue will be used in the operations of the HST to preview future updates to the pointing of the HST. False updates caused by missing stars in the current catalogue have led to spurious corrections to the pointing of the HST and a subsequent loss of observing time. The INCA consortium supplied a base catalogue of 92,560 stars extracted from the INCA databases and the TIC with $V \leq 8.5$, or $\mathrm{B} \leq 9.2$, when the visual magnitude was not available. Yang has matched those stars with numerous other catalogues and created an appendix file including stars within the magnitude limit but not in the base catalogue. A total of 28,700 stars has been added to the new FHST Catalogue to $V \leq 8.5$, or $B \leq 9.2$. Yang is also preparing an additional appendix that includes data on the duplicity and variability of each star in the catalogue.

In the absence of A. R. Klemola, R. B. Hanson and B. F. Jones, van Altena gave a report on the status of the Lick Northern Proper Motion Program. The NPM program consists of the determination of the absolute proper motions with an rms accuracy of $0.005 " / \mathrm{yr}(5 \mathrm{mas} / \mathrm{yr}$ ) for approximately 300,000 stars with respect to 70,000 faint galaxies north of declination $-23^{\circ}$. In addition, accurate positions and B, V photometry are being determined for those stars. Of the 300,000 stars, 116,000 have been selected by Klemola from over 600 references to form an Input Catalogue of Special Stars. The Yale-San Juan extension of this program to the southern hemisphere is described in the Transactions of the Commission 
24 session on the Southern Hemisphere. To date the plate surveys and measurements have been completed for 750 fields in all declinations zones outside the Milky Way from $-5^{\circ}$ to $+90^{\circ}$. The $-10^{\circ}$ zone of 50 fields is nearly complete and approximately 100 fields remain in the $-15^{\circ}$ and $-20^{\circ}$ zones. The astrometric reductions are complete for the northern sky and are in progress for the southern part of the NPM program. Tests show that the precision of a single faint NPM proper motion is 5 mas/yr while the accuracy is $2 \mathrm{mas} / \mathrm{yr}$ for the absolute zero point of an individual NPM field; the overall systematic zero-point error is $0.6 \mathrm{mas} / \mathrm{yr}$. The photometric reductions are complete for the sky north of the equator and south of $+65^{\circ}$, while they are in progress for the balance of the northern sky. Investigations related to the structure of the Galaxy utilizing the new absolute proper motions are continuing, and a comparison with other catalogues is planned. The first part of the Lick NPM Catalogue is planned to be available on magnetic tape through the data centers in mid-1992.

An abstract entitled "Progress Reports on the Kiev Observing Programs and Catalogues" was submitted by Ya. S. Yatskiv, in collaboration with N. V. Kharchenko, V. S. Kislyuk, L. K. Pakulyak, and S. P. Rybka. Yatskiv planned to describe the current status of the Photographic Four-Fold Coverage of the Northern Hemisphere (FON) as well as their study of the Main Meridional Section of the Galaxy (MEGA). The observational part of the FON project, which is carried out with six wideangle astrographs, is nearly $90 \%$ complete. The proper motions are determined using the Astrographic Catalogue for first epoch positions. As a part of the MEGA project, a general catalogue of astrometric data of 26,500 stars has been compiled and corrections to the precession constants and stellar secular parallaxes have been determined. In addition, as a part of the KSZ project, a general catalogue of absolute proper motions of 21,817 stars in 75 regions of the sky with galaxies was compiled.

N. G. Rizvanov and I. E. Tselishchev also submitted an abstract on the "Rereduction of Carte du Ciel Plates in the PPM Reference Frame in the Praesepe Region". They have rereduced 35 Paris zone plates and 10 Bordeaux zone plates of the CdC into the PPM system as a part of the establishment of a wide-angle astrometric standard region in Praesepe. The resulting catalogue, which they have compared with Fresneau's 1983 reductions, has a positional accuracy of $0.17^{\prime \prime}$.

A. R. Upgren presented the results of an investigation of the "Parallaxes of 30 Subluminous Stars and the Calibration of the Old Main Sequence", which was prepared in collaboration with H. Zhao of the Van Vleck Observatory and J. T. Lee of Yale. Using 15 newly determined parallaxes based on plates taken with the Van Vleck refractor and 15 already published, they have improved the data on the position of the old main sequence for the very metal poor stars.

\subsection{July "Developments in Space- and Ground-Based Instrumentation" chaired by J A. Hughes.}

$\mathrm{K}$. Johnston reviewed the status of the many existing and planned "Interferometers in Space and on the Ground". At the present time the Mount Wilson Interferometer is the only long baseline instrument providing astrometric observations. Precise positions for a small number of bright stars have been determined with a precision on the order of 10 mas and the orbits of a few binary stars have also been determined. Plans are underway for extended ground based arrays by the U. S. Naval Observatory for basic astrometric measurements over both wide and narrow angles, and the CHARA consortium led by Georgia State Univ. to measure binary star orbits. Many instruments are proposed for measuring the diameters of stars and imaging applications. The Mount Wilson interferometer has now measured the diameters of about fifty bright stars to one percent accuracy. The Sydney Univ. Stellar Interferometer is beginning observations of stellar diameters. The Univ. of Wyoming's IR Multiaperture Array has achieved first light. Other interferometer arrays such as CERGA's I2T and GI2T, SAO's Imaging Optical Telescope Array, and the adjunct to the VLT have achieved preliminary results or are in the planning stage. In space the concepts are as follows. Two low accuracy (1-10 mas) satellites, Lomonosov and Regatta-Astro (Space Research Institute, Moscow), have been proposed for measuring the positions of a large number of stars. Two highly accurate astrometric missions have also been proposed: POINTS (Smithsonian Astrophysical Observatory, USA) and OSI (Jet Propulsion Laboratory, USA). It is estimated that both instruments will be capable of astrometric measurements at the ten microsecond level while OSI will also be capable of imaging celestial objects.

V. K. Abalakin provided a short paper which described the Soviet AIST project (described in more detail at IAU Symposium 141), which proposes to provide a second epoch Hipparcos-like spacecraft. The main purpose of the project is to construct a fundamental system of coordinates on the 
celestial sphere with a mean accuracy of 1 mas. The goal is to obtain a density of about 10 stars per square degree, or about 400,000 stars in total, among which are included the 120,000 stars of the Hipparcos list. In addition, it is planned to fly the satellite with an epoch difference of 10 years from Hipparcos, which would yield proper motions with an accuracy of 1 mas/yr. Finally, a Tycho-like star mapper would be included to construct a catalogue of at least one million stars with an accuracy of 20 30 mas.

H. McAlister discussed "The Status of the CHARA Array", which is a multi-telescope array for very high resolution astronomy at optical wavelengths. The project is a joint effort involving astronomers from GSU/CHARA and engineers from the Georgia Tech Research Institute (GTRI) of the Georgia Institute of Technology. The Array would consist of seven 1-meter aperture telescopes configured in a non-redundant Y-shaped array contained within a circle of 400 meters diameter. This Array would provide a limiting resolution of 0.2 mas and a nominal limiting magnitude of $V=9$ to 11 depending largely upon the seeing conditions. The initial scientific goals would be the determination of fundamental astrophysical parameters of stars including masses, radii, luminosities, distances, etc. through the resolution of spectroscopic binaries and single star photospheres. The Array will also be configured so as to carry out imaging experiments for classes of objects with complex extended structures, such objects being particularly prevalent in the infrared. The feasibility of incorporating or retrofitting adaptive optics will be explored in order to make each collecting aperture fully coherent. They anticipate some funding beginning during the late fall of 1991 that will permit the further exploration of certain technical aspects such as delay lines, adaptive optics, imaging, and detectors as well as the final selection of a site for the Array. The full embarkation upon the project could then begin in late 1992 or early 1993.

Chr. de Vegt described his proposed "Plans for New Large Astrometric Telescopes" that consist of a modified Schmidt-Cassegrain layout. The design attaches the secondary directly to the achromatic corrector plate to avoid any noncircular diffraction effects. The design study is based on a 1.5 meter, F/5 layout and provides a 2.8 degree diameter, perfectly flat field, with a minimal regular third order distortion. Using standard photographic emulsions, he indicates that astrometrically measurable images of stars to magnitude 18.5 should be obtained with an exposure time of 10 minutes.

H. D. Ables presented a review of "New Developments in CCD Astrometry" at the U. S. Naval Observatory Flagstaff Station, in collaboration with C. C. Dahn and D. G. Monet. Results from the first list of 72 stars observed as a part of the USNOFS CCD parallax program were shown which demonstrated that the accuracy of the relative parallaxes ranged from 0.5 to 2.7 mas (s.e.), with a median accuracy of 1.0 mas for stars in the magnitude range $15<\mathrm{V}<20$. In addition, a new array of six CCD's with a large format $(5 \mathrm{~cm} \times 5 \mathrm{~cm})$ was shown that is currently being tested for use in the parallax program. One of the CCD's will be read out at a relatively rapid rate to avoid saturation while observing a bright parallax star, while the other five will integrate for a time sufficient to obtain an adequate signal-to-noise on the fainter reference stars. The 8-inch transit telescope is currently outfitted with a $1024 \times 1024 \mathrm{CCD}$. This system is used to obtain positions with an accuracy of 10 mas by strip scanning over lengths of many degrees. Future work is anticipated at higher accuracies with a larger aperture transit telescope using custom designed CCD arrays. Finally, a new generation precision plate measuring machine is now undergoing acceptance tests at the USNOFS which uses two cosmetically perfect $1320 \times 1035 \mathrm{CCD}$ cameras. It is planned to begin measuring the first and second epoch Palomar Sky Survey plates with an expected astrometric accuracy of $0.1 "$.

\subsection{July "Astrometry in the Southern Hemisphere" chaired by J. A. López.}

To celebrate this first General Assembly to be held in Latin America, C. E. López presented a review of "Argentinian Contributions to Astrometry" in a session that was held jointly with Commissions 8 and 26. He outlined the principal contributions to optical astrometry from the beginning, with the dedication of the Cordoba Observatory on October 24, 1871. The first catalogue, Uranometria Argentina, was observed from September 1870 to October 1871 and presented on the dedication day! Altogether, during the last 120 years, Argentinian astronomers have contributed to the improvement of the astrometric data for more than 1.3 million stars using meridian circles, astrolabes and astrographs. 
Chr. de Vegt described the Second Cape Photographic Catalogue, CPC2, which contains the positions and visual magnitudes of 276,131 stars in the approximate magnitude range $6.5<\mathrm{V}<10.5$. 5820 plates, with two 3-minute exposures, were taken in a four-fold overlap of the southern hemisphere during the period 1962 - 1972, using a newly designed 4-element lens $\left(F / 10, f=2000 \mathrm{~mm}, 4.1 \times 4.1^{\circ}\right.$ field, scale $=100^{\prime \prime} / \mathrm{mm}$ ). The plates were measured on the Royal Greenwich Observatory GALAXY machine and have been reduced using a classical plate constant method into the SRS system both in $\mathrm{B} 1950$ and J2000. A small magnitude equation in declination amounting to 75 mas from $7<\mathrm{V}<10$ has been found, but there are no apparent color effects. Quality classes in the catalogue range from 195,000 stars with positional errors of 54 mas (s.e.), 53,300 with 80 mas, and some 27,000 for identification purposes only with 200 mas errors. Preliminary proper motions have been derived for the 120,000 SAO stars in the catalogue. Final proper motions will be obtained when the recently finished FOKAT-S catalogue is available.

C. E. López and I. Platais described progress on the Yale-San Juan Southern Proper Motion (SPM) program in a report done in collaboration with T. Girard and W. van Altena. The SPM is the extension of the Lick NPM into the southern hemisphere which was described earlier. To date, of the 958 fields in the program, more than 150 second epoch fields have been repeated. The procedures for the plate measurement and reductions have been developed and catalogues of objects to be measured in an extended region around the south galactic pole are being collected. Extensive use is currently being made of the new ROE catalogue of stars and galaxies measured on the SRC Schmidt survey at Edinburgh. These catalogues are used to select stars and galaxies for measurement, in combination with PDS scans of the SPM plates which are displayed on a workstation. A continuing problem is the location of stars with poorly determined coordinates, such as variables, proper motion stars, PHL blue objects, etc. The local amateur Astronomical Society of New Haven is assisting in that part of the project. A new image centering algorithm using cubic splines has been developed and tested. It has improved convergence properties for faint stars and galaxies, but about $10-15 \%$ lower precision for the derived positions than the two-dimensional gaussian fitting method. It has been found that when scanning with certain combinations of speed and step size, the PDS microdensitometer oscillates in the direction perpendicular to the scan direction with an amplitude of several microns. This resonance can potentially lead to much lower accuracy in the derived positions when they are derived from the marginal distributions.

M. Catalan discussed the "Possibilities of Relocating the San Fernando Automatic Meridian Circle in the Southern Hemisphere". The plans are to modernize and automate the Meridian Circle so that it is similar in capabilities to the Carlsberg Automatic Meridian Circle at La Palma. If the automated Meridian Circle were then moved to the Southern Hemisphere, it would, in collaboration with the CAMC, provide full sky coverage with high accuracy instruments. The automation of the San Fernando instrument is planned to be completed in 1991 and testing will commence in 1992. Informal discussions are currently in progress with individuals at the potential sites.

J. Russell described progress that is being made in "Radio Astrometry in the Southern Hemisphere". The program for the establishment of the radio/optical reference frame link has made its greatest advances in the southern hemisphere in an international cooperative effort of astronomers from many countries, most notably Australia, South Africa, USA, Japan and Germany. They have continued to observe new sources to fill in the gaps in the distribution of sources in the southern hemisphere and to reobserve the previously catalogued sources to improve the radio positions. In the zone between $-25^{\circ}$ and $-40^{\circ}$, where there were very few sources before, there are now positions for an additional 34 with an error of roughly 1 mas. Sources south of $-40^{\circ}$ have been reobserved in both X-and S-bands to allow for ionospheric corrections. The new observations improved the accuracy to a few mas and included 33 of the 41 observed previously; other sources will be reobserved soon. Recently, an additional 51 new sources have been observed with an accuracy of 2-3 mas between the Australian antennas at Hobart and Tidbinbilla. This brings the total number of sources to 192 , close to the goal for the radio/optical link of 200 sources per hemisphere. The sources are well distributed across the sky, with the exception of the galactic plane, and especially the galactic center. The task in the southern hemisphere is made more difficult than in the north by the lack of antennas and the placement of the southern landmasses -- there are no intermediate length baselines between 1000 and $9000 \mathrm{~km}$. 
C. Anguita and M.-T. Ruiz described the status of "Parallax Observations in the Southern Hemisphere", which consists of their work at CTIO and that of P. A. Ianna at Mt. Stromlo and Siding Springs Observatories. Both of the southern parallax programs are using the methods developed by Monet and Dahn for the determination of trigonometric parallaxes with CCD detectors. Ianna at the Siding Springs Observatory has initiated a CCD parallax program with the one-meter reflector that concentrates on astrophysically interesting stars and faint high proper motion stars. In addition, he is also continuing with the more traditional photgraphic parallax program with the old Yale refractor at Mt. Stromlo, where the concentration is on high proper motion stars and members of the Hyades cluster. Anguita and Ruiz are using the CTIO $1.5-\mathrm{m}$ telescope with the $\mathrm{f} / 13$ Cassegrain focus and a CCD detector. The program stars are selected from the LHS Catalogue and from a survey by Ruiz and her collaborators. Most of the stars have turned out to be sub-dwarfs, cold white dwarfs and red dwarfs. The precision of the $x, y$ image positions, which were obtained using the algorithms of DAOPHOT software package, is on the average 6 mas $(0.02$ pixel) in the range 2,000 to 30,000 electron counts, with a sky background of about 800 electron counts. The precision deteriorates exponentially below 2000 electron counts. A comparison of the CTIO parallax of vB10 with the U.S. Naval Observatory's CCD parallax for the same star shows excellent agreement. The current program is continuing with a sample of about 50 southern faint high proper motion stars.

H. McAlister described the slowly improving situation of "Double Star Observations in the Southern Hemisphere". Observers of southern double stars have included some of the greatest names in the field (Herschel, Rossiter, van den Bos, Finsen), but their programs of discovery and measurement have been followed by long periods of inactivity. In recent years, southern double star astrometry has largely been conducted by micrometer observers based in the north who make occasional trips to southern observatories. Many systems discovered in the south have not been adequately observed over the years and hence the elucidation of their orbital motions has been delayed. The GSU/CHARA program of binary star speckle interferometry has been conducted from Kitt Peak since 1975 with observing runs scheduled two to three times per year. This program was extended to full sky coverage in 1988 with twice yearly trips to CTIO where the GSU/CHARA speckle camera system is used on the 4-m telescope. Several thousand measures have been reduced to date, and the first series included the discoveries of five new southern binaries including an additional close component to HR 6027. It is their intention to maintain this southern hemisphere speckle program as long as resources and access to telescope time permit.

\subsection{July "Linking the Optical and Radio Reference Frames" chaired by T. E. Corbin.}

This session was held jointly with Commissions 8 and 40. The reports can be found in the Transactions of Commission 8.

\subsection{July "Optical Reference Frames and Catalogues: Fainter Extension of the Optical Frame" chaired by L. Morrison.}

This session is a continuation of a Joint Meeting with Commission 8 that bears the same title, but was chaired by $\mathrm{K}$. Johnston. The reports from that first session can be found in the Transactions of Commission 8.

Chr. de Vegt reported on joint work with J. A. Hughes concerning "The USNO/Hamburg Global Astrometric Faint Star Catalogue Project". They propose that a high precision astrometric catalogue using a modern astrograph be established with a limiting magnitude to at least $V=14$ in both hemispheres. The standard four-fold plate overlap procedure combined with the Hipparcos reference frame and a global block adjustment should yield a positional accuracy of 0.05 " and a global system accuracy of $0.01 "$. The observing program should take less than three years, and with parallel plate measuring, the whole project could be completed in five years. This proposed catalogue would provide for the first time a homogeneous, global new epoch for the final reduction of the Astrographic Catalogue and a rigorous basis for the astrometric calibration of all large Schmidt sky surveys.

L. Helmer described the "Candidate Catalogue for a Faint Extension of the Fundamental Reference Frame". This Catalogue is intended to create a net of faint reference stars in the magnitude range $11.5<\mathrm{V}<12.0$, which would be useful for photographic work. At the Copenhagen University Observatory, stars in the northern sky have been selected at a density of about one per square degree, mainly from the Astrographic Catalogue. The positions have been transformed to the FK5 system and 
proper motions derived where possible. The candidate stars are proposed for reobserving on automatic meridian circles and have been included in the program for the CAMC on La Palma. The first results from the CAMC have been compared with observations made with the Bordeaux automatic meridian circle and systematic errors do not exceed 0.02 ", with a success rate close to $98.5 \%$. The selection is now complete from declinations $+90^{\circ}$ through $-52^{\circ}$, while the observations are $31 \%$ complete.

I. Platais reported on "The Yale-San Juan Plans for a Faint Secondary Reference Frame" in collaboration with T. Girard, C. E. Lopez and W. van Altena. In previous papers, proposals have been presented to extend the faint reference frame to $\mathrm{V}<14$, however there is still a need for fainter reference stars for the large reflectors and deep Schmidt photographs. These instruments are used to determine positions of stars, galaxies, QSO's and other objects for the linkage of the radio and optical frames and for multi-object spectrographs and other instruments. Using plates from the Southern Proper Motion program, a network of faint secondary standards south of declination - $17^{\circ}$ is being determined from approximately the 12 th to the 18 th magnitudes with a density of 10 to 15 stars per square degree at the faint end of the sequence. The positional accuracy (at the mean epoch of 1980) of the equatorial coordinates for the faint standards is hoped to be about $0.10^{\prime \prime}$, while the accuracy of the absolute proper motions should be about 4 to 5 mas/yr.

C. Turon highlighted the "Hipparcos Contributions" to the establishment of a faint reference frame and noted that many of the details could be found in the proceedings of the Joint Discussion on Hipparcos in this volume. The faint part of the program consists of 2400 stars fainter than V=11 and about 30,000 stars in the range $9<\mathrm{V}<11$. T. Corbin has selected stars for the extension of the fundamental system from the faintest Hipparcos stars, while L. Morrison has been responsible for the next brighter group. Approximately 1000 IRS stars are not being observed by Hipparcos and C. Smith is working on the addition, or replacement, of those IRS stars by candidate Hipparcos stars. In addition, the list of radio-stars and "link" stars observed by Hipparcos, that are close to QSO's, have been distributed to the relevant individuals in the HST project and to the ground-based observers.

P. Hemenway outlined the "Status of the Hubble Space Telescope for Making Observations Towards Linking the Hipparcos and Extragalactic Reference Frames" on behalf of the Space Telescope Astrometry Team. The status for astrometric measurement of the Fine Guidance Sensors (FGS) of the Hubble Space Telescope, including a description of their operation, was given at the IAU Colloquium 127. Since that report, measurements of about 25 stars at each of 10 pointings have been reduced for a preliminary Optical Field Angle Distortion (OFAD) calibration. The observations were made with the guiding system in Coarse Track and the astrometer FGS operating in Fine Lock. The results show residuals from the overlapping plate solution of 3-4 mas. Because the spacecraft stability was poorer at that time, the accuracy in the future may be as good as originally expected, i.e., 2 mas rms per observation. Once the final collimation position for the secondary mirror is determined, probably in early November 1991, it should then be possible to start the astrometric calibration and science observations. Due to the reduced amplitude of the FGSs transfer functions, it may be necessary to restrict observations to objects brighter than 15 th mag. In that case, it will be necessary to extend the observations for the Hipparcos Link to bright QSOs and BL Lacs, which may be radio-quiet. As a result, the emphasis would be on determining the rotation of the Hipparcos instrumental system with respect to the extragalactic frame, which with 15 objects will be at the 2 mas/yr level. If it is possible to obtain the proper motions for more objects, and over a longer time base, the expected accuracy may be as good as $0.5 \mathrm{mas} / \mathrm{yr}$ to the system rotation.

F. Chollet and N. Capitaine described a new procedure for computing the apparent places of the stars referred to the intermediate frame. This frame is linked to the nonrotating origin for estimating the Earth's rotation for astrometric observations. The procedure is based on the matrix transformation of vector components between the geocentric celestial and terrestrial reference systems. It uses the nonrotating origin for reckoning the Earth's angle of rotation and the celestial coordinates of the Celestial ephemeris Pole to account for the effects of precession and nutation. The procedure was derived from the one used to compute the apparent positions of stars for astrolabe reductions using the FK5 and can be applied to the Hipparcos frame. 